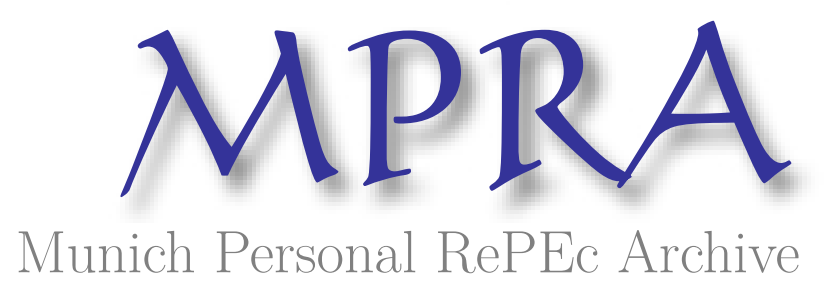

\title{
On the comparison of alternative specifications for money demand: The case of extremely low interest rate regimes in Japan
}

Nakashima, Kiyotaka and Saito, Makoto

Konan University

2 April 2012

Online at https://mpra.ub.uni-muenchen.de/70765/

MPRA Paper No. 70765, posted 16 Apr 2016 05:32 UTC 


\title{
On the comparison of alternative specifications for money demand: The case of extremely low interest rate regimes in Japan
}

\author{
KIYOTAKA NAKASHIMA *† \\ KonAn University
}

\author{
MAкото SAITO \\ Hitotsubashi UNIVERSITY
}

April, 2012

\begin{abstract}
Using Japanese money market data, this paper compares the predictive ability of the log-log specification with infinite elasticity at a zero interest rate and the semilog specification with a one time switch from moderate to relatively high semielasticity at annual interest rates less than $0.5 \%$. We find that the latter specification dominates the former in terms of predictive ability for the extremely low interest rate regime (the period between 1999 and 2006) because under the former the semielasticity is excessively sensitive to slight changes in interest rates. We find that interest rate semielasticity has remained stable at a high level since the mid-1990s.
\end{abstract}

JEL classification: E31, E41, E52.

Keywords: money demand, zero interest rate policy, structural break, bootstrap, predictive ability comparison.

* Correspondence to: Kiyotaka Nakashima, Faculty of Economics, Konan University, Okamoto 8-9-1, Higashinada, Kobe, Zip 658-8501, Japan, e-mail: kiyotaka@center.konan-u.ac.jp, fax: +81-078-452-5058.

$\dagger$ The authors would like to thank Laurence Ball, Paul Beaudry, R. Anton Braun, Hiroshi Fujiki, Fumio Hayashi, Yasushi Iwamoto, Ryuzo Miyao, Hiroshi Nakaota, Masao Ogaki, Shinichi Sakata, Masahiko Shibamoto, Mototsugu Shintani, Shigenori Shiratsuka, Yosuke Takeda, two anonymous referees, participants at Sophia University, Hosei University, Osaka University, and Keio University, and attendees at the CIRJE-TCER Macroeconomics Conference for helpful comments and suggestions. The authors also gratefully acknowledge a Grant-in-Aid from the Ministry of Education and Science of the Government of Japan. 
1. Introduction Exploring a better specification to describe money demand behavior at near-zero interest rates is important empirical work from both normative and positive viewpoints. From a normative viewpoint, welfare costs of inflation substantially depend on which specification we use when nominal interest rates are extremely low (e.g., Lucas (2000), Ireland (2009)). From a positive viewpoint, a major issue when specifying money demand functions involves how we should describe the phenomenon of the liquidity trap in relation to the interest rate semielasticity of the demand for money (e.g., Miyao (2002), Nakashima and Saito (2009)). ${ }^{1}$

Using Japanese money market data, this paper investigates the empirical plausibility of the log-log specification to characterize the money demand function observed during a regime with extremely low interest rates. ${ }^{2}$

The log-log specification has two major features. First, high semielasticity at near-zero interest rates can be captured using a simple linear representation. In this framework, real money balances can become arbitrarily large without reaching the finite satiation point, at or above which the marginal utility of real money balances is zero. Second, semielasticity with respect to interest rates is excessively sensitive to slight changes in interest rates in the neighborhood of zero rates. By exploiting the first feature, many studies, including those of Miyao (2002), Fujiki and Watanabe (2004), and Bae, Kakkar, and Ogaki (2006), have employed the log-log specification to characterize Japanese money demand functions for regimes with extremely low interest rates. ${ }^{3}$

As an alternative, this paper explores whether the second feature of the log-log specification is compatible with the shape of money demand functions. Even during the extremely

\footnotetext{
${ }^{1}$ In the context of modern macroeconomic analysis with New Keynesian models, there is a controversy regarding whether money demand relations matter or not (e.g., McCallum (2008), Nelson (2008), Woodford (2008)). For the Japanese economy in the 1990s, Canova and Tobias (2010) pointed out that models without money cannot sufficiently explain cyclical fluctuations in output and inflation.

2 From September 1995, the Bank of Japan (hereafter, BOJ) developed a low interest rate policy with the overnight call rate (interbank rate) guided below 0.5\%. In February 1999, the BOJ adopted its so-called zero interest rate policy where the call rate was set close to zero. Following a temporary lifting of the zero interest rate policy, the BOJ adopted a quantity-easing policy in March 2001. Within this framework, call rates averaged less than $0.03 \%$. The BOJ terminated the quantity-easing policy in April 2006, and the zero interest rate policy in July 2006, and accordingly maintained the overnight call rate at around $0.5 \%$. Since December 2008, the BOJ has lowered the call rate below $0.1 \%$.

${ }^{3}$ Bae and de Jong (2007) employed the log-log specification to describe US money demand behavior for the period of World War II, during which interest rates remained near zero.
} 
low interest rate regime, the call rate (interbank rate), as the principal policy instrument, fluctuated in the neighborhood of zero. Casual observation also reveals that money demand did not respond very sensitively to slight changes in interest rates during the low interest rate regime. As shown in Figure 1, the money stock (M1) relative to nominal GDP has expanded substantially since the mid-1990s, but was still quite stable despite small but frequent changes in interest rates near zero during the 2000s.

To illuminate the second feature, we adopt as an alternative specification the semilog specification with a onetime switch from moderate to relatively high semielasticity at nearzero rates. Under this alternative, semielasticity is large, but constant over time during the extremely low interest rate regime. Hereafter, we refer to the above specification as the joined semilog specification in the sense that the money demand functions are characterized by a combination of two linear functions with different degrees of semielasticity. ${ }^{4}$ Unlike the log-log specification, the joined semilog specification can define the finite satiation point at the zero interest rate bound.

To estimate the joined semilog specification, we employ the econometric tests for a structural break proposed by Hansen (1992) and Kuo (1998). Given that short-term nominal interest rates declined almost monotonically from the early 1990s to the early 2000s, tests for a structural break with respect to interest rate semielasticity allow us to identify the nominal interest rate below which more interest-elastic money demand emerges by detecting the point of change in the interest rate semielasticity.

In this paper, we also deal carefully with the small-sample problems associated with both the structural break tests and model selection. For the structural break test, Gregory, Nason, and Watt (1996) point out that the asymptotic distribution constructed by Hansen (1992) may be subject to serious small-sample bias. We avoid this problem by using the sieve bootstrap procedure proposed by Chang et al. (2006); the parametric bootstrap in Chang et al. (2006) provides a practical means of substantially reducing the small-

\footnotetext{
${ }^{4}$ Using time-series data from developed countries, a number of empirical studies confirm that interest rate semielasticity and income elasticity are stable over time using a semilog specification for money demand (see Lucas (1988), Stock and Watson (1993), and Ball (2001) for references). Using Japanese money market data before the mid-1990s, Miyao (1998) also found that the semielasticity of demand for M1 was quite stable when the linear semilog specification was employed.
} 
sample biases in the cointegrating regression. Further, we base our model selection not on a conventional measure, such as the sum of squared errors (hereafter SSE), but rather the bootstrap probability. This is because, when using conventional measures, a model may be designated as optimal by chance when the prediction period used for the performance comparison is not sufficiently long. In contrast, the bootstrap probability measures the proportion of time during which one model outperforms the other among the simulated outcomes. In computing the bootstrap probability, we again employ the sieve bootstrap procedure used in Chang et al. (2006) to avoid small-sample problems.

We find that the joined semilog specification outperforms the log-log specification in terms of predictive ability for the regime with extremely low interest rates (or the period in Japan between 1999 and 2006). That is, during this particular regime, the semielasticity was not so sensitive to slight changes in interest rates, and while large, was constant. Our findings imply that real money balances can reach the finite satiation point at the zero interest rate bound.

The paper is organized as follows. Section 2 introduces the semilog and log-log models for money demand in Japan and discusses the estimation and test results. Section 3 offers a conclusion. The Appendix describes the bootstrap procedures for constructing the critical values and conducting the forecast evaluation.

2. Estimation and Test Results In this section, we specify and estimate the money demand functions using the semilog, log-log, and joined semilog specifications. In so doing, we take into consideration the possibility that interest rate semielasticity became rather large under the extremely low interest rate regime. We then empirically investigate which model outperforms the others in terms of predictive ability.

2.1. Specification of Japanese money demand To model the money demand functions, we consider the following specifications:

$$
\begin{aligned}
& m_{t}-p_{t}=\text { constant }+\alpha y_{t}+\beta i_{t}+\epsilon_{t}, \\
& m_{t}-p_{t}=\text { constant }+\gamma y_{t}+\theta \log i_{t}+\xi_{t}
\end{aligned}
$$


where $\epsilon$ and $\xi$ designate stochastic shocks to money demand. Equation (1) represents the semilog specification where $\alpha$ and $\beta$ denote income elasticity and interest rate semielasticity, respectively. The satiation point of real money balances implied by Equation (1) is equal to $\exp ($ constant) when $\alpha$ is fixed at unity and thereby real money balances are expressed as a fraction of real income. Equation (2) characterizes the log-log specification where $\gamma$ and $\theta$ denote income and the interest rate elasticity, respectively. The interest rate semielasticity implied by Equation (2) is equal to $\theta / i_{t}$.

In addition, we consider the joined semilog specification in which two semilog specifications are joined to each other once it is statistically confirmed that $\beta$ increases significantly for the period with extremely low interest rates. That is, if there is a onetime structural break in the parameters, including $\beta$ in Equation (1), then one semilog specification is joined to another with a different set of parameters.

The test and estimation procedures are as follows. Employing a method proposed by Gregory and Hansen (1996), we first test for the absence of a cointegrating relationship in Equations (1) and (2) against the presence of cointegration with a possible structural break. Unfortunately, and as emphasized in Gregory and Hansen (1996), while their test is powerful for rejecting the absence of cointegration, it cannot test for parameter constancy and is unable to identify the structural breakpoint. Hence, when we have rejected the absence of cointegration for the two equations, we employ the tests proposed by Hansen (1992) and Kuo (1998) to test for parameter constancy and to identify the structural breakpoint.

To test for the presence of a structural break under cointegration, we choose the test proposed by Hansen (1992) for a pure structural change, where constancy in the entire set of parameters is tested against parameter instability. The test proposed by Kuo (1998) is designed to test for a partial structural change, where constancy in subsets of parameters is examined. In both tests for structural change, the null hypothesis of cointegration with parameter stability is tested against the alternative hypothesis of cointegration with parameter instability.

However, as pointed out by Gregory, Nason, and Watt (1996), Hansen's (1992) test, which is based on the asymptotic distribution, may be subject to serious small-sample bias. 
5 Taking due consideration of this potential problem, we conduct hypothesis tests using not only the asymptotic critical value reported by Hansen (1992) and Kuo (1998), but also the critical value constructed from the sieve bootstrap proposed by Chang et al. (2006). Because the test statistics in Hansen (1992) and Kuo (1998) are asymptotically pivotal, a proper bootstrap procedure for the cointegrating regressions would provide asymptotic refinement. The sieve bootstrap procedure in Chang et al. (2006) thereby allows us to deal with the small-sample biases in the structural break tests. ${ }^{6}$

In sum, we test structural breaks using not only the critical values based on the asymptotic distribution, but also those constructed from the sieve bootstrap procedure. This means we can improve the statistical inferences for a structural break.

2.2. Data For our estimation, the sample period is August 1985 to March 1999. The principal reason for excluding the period before 1985 is that Japanese money markets were strictly regulated until the mid-1980s, and it is only since then that commercial banks and securities companies have been permitted to issue various types of money market instruments at market rates. Therefore, the money market rates were unlikely to have properly reflected market conditions before 1985. Our sample period thus starts in August 1985 when the uncollateralized call market was established. For the sample period before April 1999, nominal interest rates stayed at low levels, but were still well above zero rates during this time (see Figure 1).

As discussed extensively in Section 2.6, we specify the period between April 1999 and November 2008 as the out-of-sample period. The out-of-sample period starts in April 1999, not February when the zero interest rate policy was implemented. The reason for this is that the BOJ publicly announced a firm commitment to the zero interest rate policy in April 1999. The out-of-sample period ends in November 2008 because the BOJ

\footnotetext{
${ }^{5}$ The Monte Carlo experiment conducted by Gregory, Nason, and Watt (1996) is used to conclude that the power of Hansen's (1992) structural break test is particularly poor when the cointegrating error is nearly integrated, and that the size distortion (the tendency to reject the null too frequently) is substantial as the number of regressors becomes large and the amount of serial correlation in the cointegrating error increases.

${ }^{6}$ One major advantage of the sieve bootstrap procedure proposed by Chang et al. (2006) is that the construction of the data-generating processes can consider the contemporaneous and intertemporal correlation between the innovation in explanatory variables and the disturbance in the cointegrating regression. This consideration is essential for efficient cointegrating estimation and hypothesis testing.
} 
implemented quite different monetary operations in response to the ongoing financial crisis after December 2008.

We construct the set of monthly data as follows. We select M1, compiled and seasonally adjusted by the BOJ, as the nominal monetary aggregate because M1 reflects to a great extent the transaction demand for money. It is also common in previous empirical studies of the Japanese money demand function. ${ }^{7}$

The 2005-base consumer price index (General) constructed by the Statistics Bureau provides nominal prices, and the industrial production index documented by the Ministry of International Trade and Industry specifies real aggregate output. The overnight call rates, reported by the BOJ, are used as the nominal interest rates. All data are monthly averages. As for both the nominal monetary aggregates and industrial production, our data set is based on variables that are officially seasonally adjusted by the above agencies. The consumer price index is seasonally adjusted by the X11 method over the sample period 1970-2008.

We conduct unit root tests for each of the variables, namely, the log of real money balances for M1, the log of real output, the level of nominal interest rates (call rates), and the log of nominal interest rates, using the augmented Dickey-Fuller test (abbreviated as ADF) (Dickey and Fuller (1979)) and the Phillips-Perron test (abbreviated as Z) (Phillips and Perron (1988)). In the null hypothesis, the log of real money balances and the log of real output are specified as an $I(1)$ with drift, while nominal interest rates, and the log of nominal interest rates are specified as an $I(1)$ without drift. The unit root tests for the four variables fail to reject unit roots for the levels and reject unit roots for the first differences.

The ADF and Z tests could be biased toward accepting the null of unit roots for the log of real money balances and the log of real output because the two tests do not allow for a change in the drift term in the alternative hypothesis. Taking due consideration of the potential loss of power in the two unit root tests, we additionally conduct five unit

\footnotetext{
${ }^{7}$ As alternative monetary aggregates, we employ currency and M2+CD. We find that the estimation and test results for currency do not differ much from those later reported for M1. However, in the case of M2+CD, the Gregory and Hansen (1996) test statistics fail to reject no cointegration. The estimation and test results for the specifications including currency and $\mathrm{M} 2+\mathrm{CD}$ are available from the authors upon request.
} 
root tests: the Zivot and Andrews (1992) test, the recursive, rolling and sequential tests of Banerjee, Lumsdaine and Stock (1992), and Perron's (1997) test. For the five unit root tests, the null hypothesis is an $I(1)$ with drift, and the relevant alternative hypothesis is a trend-stationary process with a onetime break in the trend at an unknown point in time. Table 1 shows the test results for unit roots against trend-stationary with breaks. The four unit root tests other than the recursive test of Banerjee et al. (1992) fail to reject unit roots at the $5 \%$ level of significance for the log of real money balances and the log of real output. Overall, our test results indicate that the variables are first-order integrated.

2.3. Cointegration tests This subsection reports the Gregory and Hansen (1996) test results. Table 2 shows the test results for no cointegration against cointegration with breaks. The critical value based on the asymptotic distribution is available from Gregory and Hansen (1996). The construction of the critical values using the bootstrap procedure is described in the Appendix. We base our statistical inference below on the critical value computed using the bootstrap procedure.

For the semilog model (1), the Inf-ADF, Inf- $Z_{t}$, and Inf- $Z_{\alpha}$ test statistics strongly reject the null hypothesis (no cointegration) at the $1 \%$ level of significance based on the critical values of the bootstrap distribution. For the log-log model (2), the Inf- $Z_{t}$ and Inf- $Z_{\alpha}$ test statistics reject no cointegration at the $10 \%$ level of significance based on the critical values of the bootstrap distribution, although Inf-ADF test statistic does not significantly reject the null hypothesis.

The Gregory and Hansen test succeeds in rejecting no cointegration for both the semilog and log-log models. As a cross-check of the cointegrating relationships in these models, we determine cointegration rank in the cointegrating vector autoregression (VAR) methodology using the Bartlett-corrected trace test for small samples proposed by Johansen (2002). The cointegration rank test is conducted based on a three-variable VAR model: for the semilog model, it is composed of the log of real money balances, the log of real output, and the level of nominal interest rates. For the log-log model, the log of real money balances, the $\log$ of real output, and the log of nominal interest rates are included in the VAR model. We find evidence in favor of one cointegrating relationship for both the semilog and log-log 
models. ${ }^{8}$

In the following subsection, we assume that the two money demand models have cointegrating relationships with possible breaks, and conduct the structural break tests proposed by Hansen (1992) and Kuo (1998).

2.4. Structural break tests We employ the Lagrange multiplier (LM) test using the fully modified OLS estimation proposed by Phillips and Hansen (1990) for tests of cointegration with parameter stability against pure or partial structural changes. ${ }^{9}$

The first step in the test procedure for a pure structural change is to choose a breakpoint $T^{*}$. For the semilog specification (1), for example, we construct a set of time-varying parameters $\left(\alpha_{t}, \beta_{t}\right.$, constant $\left.{ }_{t}\right)$ as follows:

$$
\text { if } t<T^{*} \text {, then }\left(\alpha_{t}, \beta_{t} \text {, } \text { constant }_{t}\right)=\left(\alpha_{1}, \beta_{1}, \text { constant }_{1}\right) \text {, }
$$

and

$$
\text { if } t \geq T^{*} \text {, then }\left(\alpha_{t}, \beta_{t}, \text { constant }_{t}\right)=\left(\alpha_{2}, \beta_{2}, \text { constant }_{2}\right) \text {. }
$$

Next, we compute the LM statistics to test whether $\left(\alpha_{1}, \beta_{1}\right.$, constant $\left.{ }_{1}\right)=\left(\alpha_{2}, \beta_{2}\right.$, constant $\left._{2}\right)$. The resulting LM statistics are conventionally referred to as F-statistics. The above Fstatistics are then computed for all data points of the sample period. Following Andrews (1993), we choose a breakpoint ( $T^{*}$ in our context) in the middle-70 percent of the full sample.

There are two types of tests based on these computed F-statistics. When the timing of a structural break is treated as unknown, it is possible to adopt the Sup-F test based on the largest F-statistic. On the other hand, when the parameters $\left(\alpha_{t}, \beta_{t}\right.$, constant $\left.{ }_{t}\right)$ follow a martingale process under the alternative hypothesis, it is possible to use the Mean-F test

\footnotetext{
${ }^{8}$ The Bartlett-corrected trace statistics are obtained from the restricted-constant VAR models with three lags for the semilog models and seven lags for the log-log models. For both money demand models, a lag order was picked by the Hannan-Quinn information criterion. The computations of the trace statistics are performed using Anders Warne's program Structural VAR 0.24. The test results are available from the authors upon request.

${ }^{9}$ We also use the dynamic OLS estimation proposed by Saikkonen (1991) and Stock and Watson (1993) to conduct the pure and partial structural change tests. We confirm that the test results based on the dynamic OLS do not qualitatively differ from those obtained based on the fully modified OLS. The test results based on the dynamic OLS are available from the authors upon request.
} 
based on the average F-statistic. For a partial structural change, the above procedure is applied to a subset of $\left(\alpha_{t}, \beta_{t}\right.$, constant $\left._{t}\right)$. We consider a partial structural change to be constancy of either the intercept, income elasticity, or the interest rate semielasticity.

The above testing procedure for the pure and partial structural changes of the semilog model is wholly applicable to the log-log specification (2). For a pure structural change, we can conduct the Sup-F and Mean-F tests to determine whether a set of the parameters $(\gamma, \theta$, constant $)$ is constant over time. For a partial structural change, we apply the Sup-F and the Mean-F tests to a subset of the parameters.

Critical values based on the limiting distribution are available from Hansen (1992) for a pure structural change and Kuo (1998) for a partial structural change. For statistical inference, however, we adopt critical values constructed from the sieve bootstrap procedure in Chang et al. (2006). The construction of the critical values using the sieve bootstrap procedure is described in the Appendix.

Table 3 reports the stability test results for the semilog and log-log models. As shown in the two rows denoted (1), both the Sup-F and Mean-F tests for the semilog model indicate that there were significant pure structural changes in August 1995 with reference to the bootstrap critical values. However, for the log-log specification, neither the Sup-F nor Mean-F tests detect a significant pure structural change using the bootstrap critical values.

According to the partial structural change test based on the bootstrap critical values, the instability of the semilog model is detected only for interest rate semielasticity $(\beta)$ at the $1 \%$ level of significance, while that of the log-log model is not detected for any of the three parameters.

The above test results indicate that the pure structural change around 1995 in the semilog model could be attributed to the partial structural change of interest rate semielasticity in 1995. In contrast, the log-log model is time invariant, a finding consistent with Miyao (2002) and Nakashima (2009). ${ }^{10}$

\footnotetext{
10 Miyao (2002) provides evidence that the log-log model is stable over time using Hansen's (1992) pure structural change test. Nakashima (2009) finds no evidence that income and interest rate elasticity are state dependent using Choi and Saikkonen's (2004) linearity test. As an alternative approach, Hondroyiannis, Swamy, and Tavlas (2000) employ a random coefficient model, and find that the absolute value of interest
} 
Figure 2 plots the F-statistic for each data point, together with the $5 \%$ critical values based on both the asymptotic and bootstrap distributions for the case of the constancy of $\beta$ in the semilog model. As clearly shown, the highest F-statistic at June 1995 far exceeds the $5 \%$ critical value of the asymptotic distribution, and is above that using the bootstrap procedure. Therefore, this result implies that the constancy of the interest rate semielasticity in the semilog model is strongly rejected given an unknown breakpoint.

To additionally check parameter constancy of the semilog and log-log models in the cointegrated VAR methodology, we also conduct the fluctuation and Nyblom tests proposed by Hansen and Johansen (1999). The fluctuation test is a supremum test for the constancy of the nonzero eigenvalues of the reduced-rank matrix, while the Nyblom test provides supremum and mean test statistics for checking the constancy of cointegrating vectors. Therefore, as long as the cointegration rank is one, the Nyblom test can be regarded as a test of a pure structural change in the cointegrated VAR methodology. Table 4 reports test results for parameter constancy obtained by imposing the cointegration rank of one on the three-variable VAR models. For the semilog model, the fluctuation and Nyblom tests indicate that there were significant structural changes around 1995 according to the bootstrap critical values. ${ }^{11}$ For the log-log model, on the other hand, neither of the two tests rejects the null of parameter constancy using the bootstrap critical values. The test results for parameter constancy in the cointegrated VAR methodology are quite consistent with those for a pure structural change.

In sum, the above test results imply that the change in interest rate semielasticity contributes to the structural break in the semilog model around 1995. Therefore, the joined semilog specification is plausible in our context. On the other hand, we can regard the functional form based on the $\log -\log$ specification as time invariant. ${ }^{12}$

rate elasticity declined continuously during the low interest rate regime. Their finding, however, may be called into question because it is not clear that the random coefficient model applies when dealing with the coefficients of integrated variables.

11 Asymptotic and bootstrap critical values are from 5,000 simulations using Anders Warne's program Structural VAR 0.24. In bootstrapping, the block bootstrap procedure is performed with a block size of twelve.

12 For the second subsample period from 1995:8 to 1999:3, we conducted Hansen's (1992) test for a pure structural change using both the asymptotic and bootstrap critical values, although the reliability of the test results is not entirely assured because the number of observations available for conducting Hansen's 
2.5. Estimation results The structural break test for the semilog specification implies that two cointegrating regimes with two different degrees of interest rate semielasticity emerged around 1995. Accordingly, in estimating the joined semilog model, we assume that there are two cointegrating regimes separated by the data point with the largest Fstatistic for Hansen's (1992) pure structural change test: this breakpoint corresponds to August 1995. ${ }^{13}$ For the log-log specification (2), on the other hand, the structural break test indicates that the functional form is time invariant. Hence, we estimate the model without any sample splitting.

Using the fully modified OLS, Table 5 reports the parameter estimates obtained from the linear semilog model, the joined semilog model, and the log-log model, and their 95\% confidence intervals. ${ }^{14}$ For confidence intervals, we calculate not only the asymptotic but also the bootstrap values to deal with any small-sample problems. The construction of the bootstrap confidence intervals is described in the Appendix.

We can point out some observations about these estimated parameters. First, as expected, the joined semilog model is estimated to be much more elastic with respect to interest rates for the second period than the first period. The estimated interest rate semielasticity $(\beta)$ changes from -0.037 to -1.016 . The finding that the estimated interest rate semielasticity becomes higher around 1995 is compatible with previous studies, including Miyao (1998) and Nakashima (2009), where the estimated interest rate semielasticity

test is only 28 , and hence extremely small. For the joined semilog model in the second subsample, we have confirmed that the test for a pure structural change rejects the null of parameter constancy at the $10 \%$ level of significance using the asymptotic critical values, but it does not reject the null at the $1 \%$ level of significance using the bootstrap values. For the log-log model in the second subsample, we have confirmed that the test does not reject the null of parameter constancy at the $5 \%$ level of significance using both the asymptotic and bootstrap critical values.

13 As Hansen (1992) argues, it would be inappropriate to conclude, based only on the rejection of the Sup$\mathrm{F}$ test, that there are two cointegrating regimes separated by a data point with the largest F-statistic. This is particularly true when there is no prior knowledge of the breakpoints. Before conducting the empirical investigation, however, we have legitimate expectations that a structural break would occur around 1995 when the BOJ guided overnight call rates below $0.5 \%$, and thus implemented the low interest rate policy. Given this expectation, one of the most natural possibilities would be that a break occurred at the data point with the largest F-statistic. We pursue this possibility with the semilog specification.

${ }^{14}$ In addition to conventional linear cointegration techniques, such as the fully modified OLS or the dynamic OLS, Bae, Kakkar, and Ogaki (2006) use the nonlinear cointegration technique to estimate the loglog model for Japanese money demand, thereby dealing carefully with the statistical issue of the nonlinear transformation of interest rates as the $I(1)$ variable. Their estimation results, however, do not depend very much on the techniques used for their estimation. 
ranges from about -0.03 to about -0.1 for sample periods prior to 1995 and from about -0.5 to about -1.1 for sample periods after 1995 .

Second, the interest rate elasticity of the log-log model $(\theta)$, accompanied by a large confidence interval, is estimated to be about -0.17 . The absolute value of the estimated interest rate elasticity is then compatible with that reported by Miyao (2004) and Bae, Kakkar, and Ogaki (2006), where the estimated interest rate elasticities range from about -0.08 to about -0.18 .

Third, the income elasticity of the semilog model $(\alpha)$ is estimated to be close to unity with a small confidence interval for the first period, while its point estimate in the second period is close to unity, but is imprecise given the large confidence interval. The income elasticity of the log-log model $(\gamma)$, accompanied by a large confidence interval, is also estimated to be close to unity.

Given imprecise estimates of the coefficients on logarithmic income, Table 6 reports the parameter estimates for the semilog and log-log models in which the income elasticity is fixed at unity. The estimated interest rate semielasticity $(\beta)$ and elasticity $(\theta)$ are quite similar to those obtained without any restrictions on income elasticity. In addition, the constant term is fairly precisely estimated to be about 5.0. ${ }^{15}$

As discussed in Section 2.1, the semilog model can provide the information about the finite satiation point, that is, the minimum point of real balances at the zero interest rate bound. We estimate the satiation point in terms of Marshallian $k$ defined as the ratio of M1 to Nominal GDP. First, we obtain the logarithmic values of Marshallian $k\left(m_{t}-p_{t}-y_{t}\right.$ in Equation (1)) for the sample period from the third quarter 1995 to the first quarter 1999, which corresponds to the second period of the subsample estimation after 1995. Next, we calculate the constant term in Equation (1), or the sample average of $m_{t}-p_{t}-y_{t}-\beta i_{t}$, using the estimated interest rate semielasticity for the second period reported in Table 6 . The average is calculated to be -0.573 , and thus the satiation point is estimated to be 0.564 through $\exp (-0.573)$. The estimated satiation point exists around 2002, which is

\footnotetext{
15 We also use other methods proposed by Johansen (1991), Park (1992), and Stock and Watson (1993) to estimate the semilog and log-log models. We confirm that the estimation results based on these alternative methods are quite similar to those based on the fully modified OLS. The estimation results obtained using these alternative methods are available from the authors upon request.
} 
about one year after the BOJ adopted the quantity-easing policy in $2001 .{ }^{16}$

In sum, a single linear equation can approximate the Japanese money demand function under the log-log specification. However, under the semilog specification, two linear equations or the joined semilog can express the specification, in which the interest rate semielasticity switches from moderate to large in 1995.

2.6. Performance comparison In this subsection, we conduct a performance comparison in terms of predictive ability between the linear semilog, the joined semilog, and the log-log models. We base our model selection not on any conventional measure, such as the SSE, but rather on the bootstrap probability. This is because when using conventional measures, a model may be designated as optimal by chance when the prediction period is not sufficiently long.

The bootstrap probability measures the proportion of time during which one model outperforms the other two using the simulated outcomes. More specifically, the bootstrap probability is defined for each of the three models as follows:

$$
P_{k}=\sharp\left\{\min _{k=1,2,3} \hat{e}_{k}^{b}: b=1, \ldots, B\right\} / B,
$$

where $B$ denotes the number of bootstrap replications, $\hat{e}_{k}^{b}$ is the SSE computed for model $k$ in replication $b$, and $\sharp\{\}$ is a counter operator. By construction, $\Sigma_{k=1}^{3} P_{k}=1$ holds. ${ }^{17}$

The decision rule based on bootstrap probability, which has been widely used since Felsenstein (1985) applied it to phylogenetic tree selection, is that when the bootstrap probability of a certain model approaches one, the model concerned outperforms the other models in terms of predictive superiority. On the other hand, if the bootstrap probability of a certain model is close to zero, then either of the other models is predictively superior.

\footnotetext{
${ }^{16}$ Ireland (2009) demonstrated that the semilog specification is superior to the log-log specification in describing US money demand behavior during the period of very low interest rates from 2002 to 2004 . We find that the satiation point of US real balances implied by Ireland's (2009) estimates of the semilog specification is calculated to be about 0.17 . The calculated satiation point of US real balances is much lower than that of Japanese real balances.

17 This bootstrap-based model evaluation measure ( $P_{k}$ in our context) is referred to differently in other studies. For example, Liu and Singh (1997), Efron and Tibshirani (1998), and Shimodaira (2004) term this measure the empirical strength probability, the confidence value, and the bootstrap probability, respectively. We follow the terminology in Shimodaira (2004).
} 
In addition, when the bootstrap probability is far from either one or zero, we cannot make any strong assertion about model selection. ${ }^{18}$ We employ such a decision rule for model selection, although the statistical property of bootstrap probability in a cointegrating regression model has not yet been established. ${ }^{19}$

To calculate the bootstrap probability, we again employ the bootstrap procedure in Chang et al. (2006) with 5,000 bootstrap replications. We additionally set the number of bootstrap replications at 500,1,000,3,000 and 7,000, but respective simulations do not yield quantitatively different results reported in this subsection. The construction of the bootstrap probability is described in the Appendix.

For model selection purposes, we employ the estimation results reported in Table 6 in which the income elasticity is fixed at unity, partly because our focus is on the response of money demand to interest rates, and partly because income elasticity is estimated to be quite imprecise for all of the models.

As mentioned earlier, the in-sample period is between July 1985 and March 1999, while the out-of-sample period is between April 1999 and November 2008. For the out-of-sample period, it is noteworthy that monthly averages of the uncollateralized overnight call rates are officially reported at $0 \%$ at three data points: January 2004, April 2004 and July 2004. Consequently, the implied interest rate semielasticity $\left(\theta / i_{t}\right)$ for the log-log model takes an infinite value at the three data points. Given that the implied semielasticity becomes infinitely excessive to slight changes in nominal interest rates around a zero-value data point, the log-log model is obviously not appropriate for describing Japanese money demand behavior for the out-of-sample period because Japanese real balances were not

\footnotetext{
${ }^{18}$ Using bootstrap methods, White (2000), Hansen (2005), and Romano and Wolf (2005) test whether a particular benchmark model is significantly outperformed by alternative models. However, their tests may not be suitable for our purpose of examining relative model superiority because the rejection of the benchmark or its nonrejection may not allow us to identify the best model among competing models: known as "multiple comparisons with control." In contrast, the bootstrap probability allows us to directly evaluate the relative superiority and inferiority of competing models. We therefore employ a decision rule based on the bootstrap probability.

19 The bootstrap probability $P_{k}$ corresponds to the $\mathrm{P}$-value in testing whether model $k$ has predictive superiority over its competitors. In the multivariate normal model, the bootstrap probability approaches the exact P-value with the order $O\left(T^{-j / 2}\right)(j \geq 1)$, where the order of accuracy $j$ depends on bootstrap methods for computing the bootstrap probability (see Efron and Tibshirani (1998) and Shimodaira (2004)). To our knowledge, the statistical properties of bootstrap probability have not yet been established in cointegrating regression.
} 
volatile around the three data points (see Figure 1). We evaluate out-of-sample predictive ability below more carefully and formally using the bootstrap probability. To this end, for the three months of January 2004, April 2004 and July 2004, we utilize month-end values of the uncollateralized overnight call rates in place of monthly average values. Month-end values officially reported for the three months are $0.001 \%$ and are the same as the monthly average values before and after these months. Therefore, the replacement of data can conservatively evaluate the out-of-sample predictability of the log-log model because the implied semielasticity is not volatile around these three months. ${ }^{20}$

Tables 7 and 8 present the in-sample (Table 7) and out-of-sample (Table 8) performance comparison results. In these tables, the SSE itself is reported for the joined semilog model. For the linear semilog and log-log models, on the other hand, the difference in the SSE between the joined semilog and either of the two other models is reported. A plus sign indicates that the SSE of the linear semilog model (the log-log model) is larger than that of the joined semilog model, while a minus sign indicates the opposite. In addition, these tables report the average interest rate semielasticity, namely, the sample average of the estimated interest rate semielasticity $(\beta)$ for the joined semilog model, and that of the implied interest rate semielasticity $\left(\theta / i_{t}\right)$ for the log-log model. ${ }^{21}$ The bootstrap probabilities are in parentheses. For an evaluation of in-sample predictive ability, we also employ the leaveone-out cross-validation proposed by Stone (1974) to estimate the SSE.

We point out the following observations about the performance comparison. First, the linear semilog model carries both large positive SSEs and a small bootstrap probability, and is clearly inferior to both the joined semilog model and the log-log model for both the in-sample and out-of-sample periods. This result is compatible with Bae, Kakkar, and Ogaki (2006) who also conclude that the log-log model is superior to the linear semilog

\footnotetext{
${ }^{20}$ Month-end values of the uncollateralized overnight call rates officially reported for the months of January 2004, April 2004 and July 2004 are $0.001 \%$, but somehow monthly averages are officially reported to be $0 \%$ for these months. We confirmed that the exclusion of the three data points does not qualitatively change the empirical results of the out-of-sample performance comparison reported in this subsection.

${ }^{21}$ For example, for the full in-sample period from 1985 to 1999, the average interest rate semielasticity is defined as $T^{-1}\left(T_{1} \cdot \beta_{1}+T_{2} \cdot \beta_{2}\right)$ for the joined semilog model, and as $T^{-1} \sum_{t=1985: 7}^{1999: 3} \theta / i_{t}$ for the log-log model. $T, T_{1}$, and $T_{2}$ denote the number of observations in the full sample, the first subsample, and the second subsample, respectively. $\beta_{1}$ and $\beta_{2}$ indicate estimated interest semielasticities of the first and second subsamples.
} 
model in terms of out-of-sample predictive ability.

Second, as demonstrated in Table 7, there is no superiority in in-sample predictive ability between the joined semilog and log-log models because the bootstrap probability computed for both the full in-sample period and the two in-subsample periods takes a value close to 0.5. As shown in Figure 3, the estimated interest rate semielasticity does not differ significantly between the two models for any in-sample period except March 1999.

Third, as shown in Table 8, the joined semilog model is predictively superior to the log-log model for the full out-of-sample period from 1999 to 2008. Table 8 also reports the comparison results for the two subsamples: the period from April 1999 to June 2006 when the BOJ adopted its zero interest rate policy (the quantity-easing policy), and the period between July 2006 and November 2008 when the BOJ lifted the zero interest rate policy. Based on the subsample results, the overall superiority of the joined semilog model can be attributed to the inferiority of the $\log$ - $\log$ model for the first subsample. ${ }^{22}$

Fourth, as shown in Figure 4, the semielasticity implied by the log-log specification differs substantially from the estimated semielasticity based on the joined semilog specification for the first subsample of the out-of-sample period. Given the superiority of the joined semilog specification, this suggests that the semielasticity does not respond to small changes in interest rates as much as the log-log specification predicts, and that the log-log specification yields excess sensitivity of money demand to interest rates for near-zero rates. In other words, during the extremely low interest rate regime, interest rate semielasticity was not as volatile as implied by the log-log specification and had been relatively stable, but at a high level, since mid-1995. ${ }^{23}$

\footnotetext{
${ }^{22}$ The average interest-rate semielasticity obtained by excluding the zero-value data points (January 2004, April 2004 and July 2004) is calculated to be -63.32 for the full out-of-sample period, and -85.04 for the first out-of-subsample period.

23 The sensitivity of the level of nominal M1 stock $\left(M_{t}\right)$ to nominal interest rates, $\partial M_{t} / \partial i_{t}$, can be estimated from the estimated interest rate semielasticities $(\beta)$ and elasticities $(\theta)$; specifically, it is expressed as $\beta Y_{t} P_{t} \exp$ (constant $\left.+\beta i_{t}+\epsilon_{t}\right)$ in the semilog model with unitary income elasticity, and as $\theta \exp ($ constant + $\left.\xi_{t}\right) Y_{t} P_{t} i_{t}^{\theta-1}$ in the $\log$-log model with unitary income elasticity, where $Y_{t}$ and $P_{t}$ denote the levels of real output (industrial production index) and nominal price (consumer price index). We confirmed that the behavior of estimated sensitivities of the joined semilog and log-log models is substantially the same as that of estimated semielasticities of the two models reported in this subsection. The estimated sensitivities are available from the authors upon request.
} 
3. Conclusion Using a predictive ability comparison between the log-log specification and the joined semilog specification, we conclude that the estimated interest rate semielasticity became extremely large when call rates (interbank rates) were below $0.5 \%$ in the mid-1990s, and that it has been stable, but at a rather high level, since this time. We find that the log-log specification successfully captures the former dimension, but fails to fit the latter because the implied semielasticity is too sensitive to small changes in interest rates that are near-zero rates. On the other hand, the joined semilog specification with a onetime switch from moderate to relatively high semielasticity at interest rates below $0.5 \%$ succeeds in simultaneously accommodating these two aspects. Our findings suggest that when nominal interest rates are near zero, real money balances are not as volatile as the log-log specification predicts, but are instead stable around the finite satiation point, albeit at a rather high level.

Our conclusions involve only positive analysis of the money demand function. As discussed in the Introduction, the welfare cost of inflation would be the most important normative implication. Exploring the normative implications of money demand specifications in the context of each theoretical background remains a critical task for our future research. 24

Appendix: Bootstrap Procedures This Appendix describes the bootstrap procedures for constructing the critical values in Tables 2 and 3, the confidence intervals in Tables 5 and 6 , and the forecast evaluation in Tables 7 and 8 .

Our bootstrap procedures described in this Appendix and the results reported in the main text are based on 5,000 bootstrap replications. We additionally set the number of bootstrap replications at 500,1,000,3,000 and 7,000, but these simulations do not yield quantitatively different results from those reported in the main text.

A1. Bootstrap procedures for the cointegration test We compute the bootstrap distributions and the corresponding critical values of the test statistics Inf-ADF, Inf- $Z_{t}$, and Inf- $Z_{\alpha}$ for the cointegration test in Gregory and Hansen (1996) in the following way.

\footnotetext{
${ }^{24}$ As of the time of writing, we have found that the welfare costs of inflation estimated with the joined semilog specification would be substantially lower than those with the log-log specification.
} 
1. Estimate the semilog money demand function (1) using a full sample of size $n=163$ by OLS to obtain the fitted residuals $\left\{\epsilon_{1}, \epsilon_{2}, \ldots, \epsilon_{n}\right\}$.

2. Define $\left\{u_{1}, u_{2}, \ldots, u_{n}\right\}$, where $u_{j}=\Delta \epsilon_{j}$, assuming that the stochastic disturbance $\epsilon_{t}$ follows a random work process under the null hypothesis of no cointegration, and sample $\left\{\hat{u}_{1}, \hat{u}_{2}, \ldots, \hat{u}_{n}\right\}$ randomly with replacement from the centered residuals $\left\{u_{j}-\right.$ $\bar{u}: j=1, \ldots, n\}$, where $\bar{u}=n^{-1} \sum_{j=1}^{n} u_{j}$.

3. Obtain a bootstrap sample $\left\{\hat{\epsilon}_{1}, \hat{\epsilon}_{2}, \ldots, \hat{\epsilon}_{n}\right\}$ for the stochastic disturbance $\epsilon_{t}$ by integrating $\left\{\hat{u}_{j}\right\}$, that is, $\hat{\epsilon}_{j}=\epsilon_{0}+\sum_{k=1}^{j} \hat{u}_{k}$, where $\epsilon_{0}$ indicates the initial value of the residuals $\left\{\epsilon_{j}\right\}$.

4. Generate a bootstrap sample $\left\{\hat{m}_{j}-\hat{p}_{j}: j=1, \ldots, n\right\}$ of a real money balance by substituting the bootstrap residuals $\left\{\hat{\epsilon}_{j}\right\}$ as well as the observed explanatory variables $\left\{y_{j}, i_{j}: j=1, \ldots, n\right\}$ into the OLS-estimated money demand function.

5. Apply Gregory and Hansen's (1996) test to each bootstrap sample $\left\{\hat{m}_{j}-\hat{p}_{j}, y_{j}, i_{j}\right.$ : $j=1, \ldots, n\}$, and repeat this procedure 5,000 times to compute the bootstrap distributions of the Inf-ADF, Inf- $Z_{t}$, and Inf- $Z_{\alpha}$ statistics. Set the bootstrap $\alpha$-level critical values equal to the $1-\alpha$ quantiles of the bootstrap distributions.

6. These bootstrap procedures are thoroughly applicable to the log-log money demand function (2).

A2. Bootstrap procedures for the structural change tests For efficient estimation and hypothesis testing, Chang et al. (2006) developed a sieve bootstrap method. The sieve bootstrap method suggests the use of a finite-order vector autoregression (VAR) for specifying the structure of the contemporaneous and intertemporal correlation between the innovation in explanatory variables and the disturbance in a cointegrating regression. Employing the VAR-based sieve bootstrap method, we compute both bootstrap distributions and critical values of Sup-F and Mean-F in the following way.

1. Estimate the semilog money demand function (1) using a full sample of size $n=163$ by fully modified OLS to obtain the fitted residuals $\left\{\epsilon_{1}, \epsilon_{2}, \ldots, \epsilon_{n}\right\}$. 
2. Let $\left\{y_{j}, i_{j}: j=1, \ldots, n\right\}$ denote the observed explanatory variables, and define $\left\{\omega_{j}=\left(\epsilon_{j}, v_{j}^{y}, v_{j}^{i}\right)^{\prime}: j=1, \ldots, n\right\}$, where $v_{j}^{y}=\Delta y_{j}$ and $v_{j}^{i}=\Delta i_{j}$.

3. Suppose that the DGP of $\left\{\omega_{j}\right\}$ is given by the $q$-th order VAR $\omega_{t}=\sum_{k=1}^{q} \Phi_{k} \omega_{t-k}+\eta_{t}$, and estimate the VAR using $\left\{\omega_{j}\right\}$ by OLS to obtain the estimates $\left\{\Phi_{1}, \Phi_{2}, \ldots, \Phi_{q}\right\}$ and the fitted residuals $\left\{\eta_{1}, \eta_{2}, \ldots, \eta_{n}\right\}$. The order $q$ is chosen using the Schwartz information criterion.

4. Sample $\left\{\hat{\eta}_{1}, \hat{\eta}_{2}, \ldots, \hat{\eta}_{n}\right\}$ randomly with replacement from the centered VAR residuals $\left\{\eta_{j}-\bar{\eta}: j=1, \ldots, n\right\}$, where $\bar{\eta}=n^{-1} \sum_{j=1}^{n} \eta_{j}$, and construct a bootstrap sample $\left\{\hat{\omega}_{j}=\left(\hat{\epsilon}_{j}, \hat{v}_{j}^{y}, \hat{v}_{j}^{i}\right)^{\prime}: j=1, \ldots, n\right\}$ recursively using $\hat{\omega}_{j}=\sum_{k=1}^{q} \Phi_{k} \hat{\omega}_{j-k}+\hat{\eta}_{j}$ given the initial values $\left\{\hat{\omega}_{j}=\omega_{j}: j=0, \ldots, 1-q\right\}$. Steps 3 and 4 above correspond to the VAR-based sieve bootstrap method proposed by Chang et al. (2006).

5. Obtain a bootstrap sample $\left\{\hat{y}_{j}, \hat{\imath}_{j}: j=1, \ldots, n\right\}$ of the explanatory variables by integrating $\left\{\hat{v}_{j}^{y}, \hat{v}_{j}^{i}\right\}$, that is, $\hat{y}_{j}=j \cdot \mu^{y}+y_{0}+\sum_{k=1}^{j} \hat{v}_{k}^{y}$ and $\hat{\imath}_{j}=i_{0}+\sum_{k=1}^{j} \hat{v}_{k}^{i}$, where $\mu^{y}$ indicates the drift term of $y_{t}$ estimated from $\mu^{y}=n^{-1} \sum_{j=1}^{n} \Delta y_{j}$, and a pair $\left(y_{0}, i_{0}\right)$ indicates the initial value of $\left\{y_{j}, i_{j}\right\}$.

6. Generate a bootstrap sample $\left\{\hat{m}_{j}-\hat{p}_{j}: j=1, \ldots, n\right\}$ of a real money balance by substituting the bootstrap residuals $\left\{\hat{\epsilon}_{j}\right\}$ as well as the bootstrap explanatory variables $\left\{\hat{y}_{j}, \hat{\imath}_{j}\right\}$ into the money demand function estimated by fully modified OLS.

7. Apply Hansen's (1992) test for pure structural changes and Kuo's (1998) test for partial structural changes to each set of the bootstrap sample $\left\{\hat{m}_{j}-\hat{p}_{j}, \hat{y}_{j}, \hat{\imath}_{j}\right\}$, and repeat this procedure 5,000 times to compute the bootstrap distributions of the Sup$\mathrm{F}$ and Mean-F statistics. Set the bootstrap $\alpha$-level critical values equal to the $1-\alpha$ quantiles of the bootstrap distributions.

8. These bootstrap procedures are thoroughly applicable to the log-log money demand function (2).

A3. Bootstrap confidence intervals To obtain bootstrap confidence intervals, we merely alter Step 7 in the bootstrap procedure for the structural change tests: we calculate 
the confidence intervals by not applying the structural change tests but, instead, the fully modified OLS to the bootstrap sample. According to Tables 5 and 6 , while the estimated confidence intervals are somewhat larger than those based on the asymptotic distribution, the sign and significance of the estimated parameters do not change substantially.

A4. Bootstrap procedures for comparison of predictive ability To conduct a predictive ability comparison, we alter the bootstrap procedure for the structural break tests as follows. First, we define the sum of squared errors (SSE) as $\left\{e_{k}: k=1,2,3\right\}$, where $e_{k}$ indicates the SSE for the joined semilog, linear semilog, and log-log models. Tables 7 and 8 report the SSE of each model as a statistic.

Next, in Step 7, we obtain parameter estimates by applying the fully modified OLS to the bootstrap sample. Using the estimated parameter, we generate fitted residuals in a prediction period, and then calculate the bootstrap $\operatorname{SSE}\left\{\hat{e}_{k}^{b}: b=1, \ldots, B\right\}$ for the three models. In each replication $b$, the bootstrap SSE for the best model is defined as $\left\{\min _{k=1,2,3} \hat{e}_{k}^{b}: b=1, \ldots, B\right\}$. We repeat this procedure $B=5,000$ times to obtain the bootstrap probability $P_{k}$, that is, the proportion of time during which one model outperforms the other two models. 


\section{REFERENCES}

[1] Andrews, Donald W. K., 1993, "Tests for parameter instability and structural change with unknown change point," Econometrica 61, 821-856.

[2] Bae, Youngsoo, and Robert M. de Jong, 2007, "Money demand function estimation by nonlinear cointegration," Journal of Applied Econometrics 22, 767-793.

[3] Bae, Youngsoo, Vikas Kakkar, and Masao Ogaki, 2006, "Money demand in Japan and nonlinear cointegration," Journal of Money, Credit and Banking 38, 1659-1668.

[4] Ball, Laurence, 2001, "Another look at long-run money demand," Journal of Monetary Economics 47, 31-44.

[5] Banerjee, Anindya, Robin L. Lumsdaine, and James H. Stock, 1992, "Recursive and sequential tests of the unit-root and trend-break hypothesis: Theory and international evidence," Journal of Business and Economic Statistics 10, 217-287.

[6] Canova, Fabio, and Menz Tobias, 2010, "Japan's lost decade: Does money have a role?" Journal of the Japanese and International Economies 24, 178-195.

[7] Chang, Yoosoon, Joon Y. Park, and Kevin Song, 2006, "Bootstrapping cointegrating regressions," Journal of Econometrics 133, 703-739.

[8] Choi, In, and Pentti Saikkonen, 2004, "Testing linearity in cointegrating smooth transition regressions," Econometrics Journal 7, 341-365.

[9] Dickey, David A., and Wayne A. Fuller, 1979, "Distribution of the estimators for autoregressive time series with a unit root," Journal of the American Statistical Association $74,427-431$.

[10] Efron, Bradley, and Robert Tibshirani, 1998, "The problem of regions," The Annals of Statistics 26, 1687-1718.

[11] Felsenstein, Joseph, 1985, "Confidence limits on phylogenies: An approach using the bootstrap," Evolution 39, 783-791. 
[12] Fujiki, Hiroshi, and Kiyoshi Watanabe, 2004, "Japanese demand for narrow monetary aggregate in the 90s: Time series versus cross-sectional evidence from Japan," Monetary and Economic Studies 22, 47-77.

[13] Gregory, Allan W., and Bruce E. Hansen, 1996, "Residual-based tests for cointegration in models with regime shifts," Journal of Econometrics 70, 99-126.

[14] Gregory, Allan W., James M. Nason, and David G. Watt, 1996, "Testing for structural breaks in cointegrated relationships," Journal of Econometrics 71, 321-341.

[15] Hansen, Bruce E., 1992, "Tests for parameter instability in regressions analysis with I(1) processes," Journal of Business and Economic Statistics 10, 321-335.

[16] Hansen, Henrik, and S $\phi r e n$ Johansen, 1999. "Some tests for parameter constancy in cointegrated VAR-models," Econometrics Journal 2, 306-333.

[17] Hansen, Peter R., 2005, "A test for superior predictive ability," Journal of Business and Economic Statistics 23, 365-380.

[18] Hondroyiannis, George, P. A. V. B. Swamy, and George S. Tavlas, 2000, "Is the Japanese economy in a liquidity trap?" Economics Letters 66, 17-23.

[19] Ireland, Peter P., 2009, "On the welfare cost of inflation and the recent behavior of money demand," American Economic Review 99, 1040-1052.

[20] Johansen, S $\phi$ ren, 1991, "Estimation and hypothesis testing of cointegration vectors in Gaussian vector autoregressive models," Econometrica 59, 1551-1580.

[21] Johansen, S $\phi$ ren, 2002, "A small sample correction of the test for cointegrating rank in the vector autoregression model," Econometrica 70, 1929-1961.

[22] Kuo, Biing-Shen, 1998, "Test for partial parameter instability in regressions with I(1) processes," Journal of Econometrics 86, 337-368.

[23] Liu, Regina Y., and Kesar Singh, 1997, "Notions of limiting P values based on data depth and bootstrap," Journal of the American Statistical Association 92, 266-277. 
[24] Lucas, Robert E., 1988, "Money demand in the United States: A quantitative review," Carnegie-Rochester Conference Series on Public Policy 29, 137-168.

[25] Lucas, Robert E., 2000, "Inflation and welfare," Econometrica 68, 247-274.

[26] McCallum, Bennett T., 2008, "How important is money in the conduct of monetary policy? A comment," Journal of Money, Credit and Banking 40, 1783-1790.

[27] Miyao, Ryuzo, 1998, "Note on the stability of long-run money demand: Is the interest elasticity really constant?" Discussion Paper Series, No. 94, Research Institute for Economics and Business Administration, Kobe University.

[28] Miyao, Ryuzo, 2002, "Liquidity traps and the stability of money demand: Is Japan really trapped at the zero bound?" Discussion Paper Series, No. 127, Research Institute for Economics and Business Administration, Kobe University.

[29] Nakashima, Kiyotaka, 2009, "An extremely low interest rate policy and the shape of the Japanese money demand function: A nonlinear cointegration approach," Macroeconomic Dynamics 13, 553-579.

[30] Nakashima, Kiyotaka, and Makoto Saito, 2009, "On empirical implications of highly interest-elastic money demand: A note," Hitotsubashi Journal of Economics 50, 29-34.

[31] Nelson, Edward, 2008, "Why money growth determines inflation in the long run: Answering the Woodford Critique," Journal of Money, Credit and Banking 40, 17911814.

[32] Park, Joon Y., 1992, "Canonical cointegration regressions," Econometrica 60, 119-143.

[33] Perron, Pierre, 1997, "Further evidence on breaking trend functions in macroeconomic variables," Journal of Econometrics 80, 355-385.

[34] Phillips, Peter C. B., and Bruce E. Hansen, 1990, "Statistical inference in instrumental variables regression with I(1) processes," Review of Economic Studies 57, 99-125.

[35] Phillips, Peter C. B., and Pierre Perron, 1988, "Testing for a unit root in time series regression," Biometrika 75, 335-346. 
[36] Romano, Joseph P., and Michael Wolf, 2005, "Stepwise multiple testing as formalized data snooping," Econometrica 73, 1237-1282.

[37] Saikkonen, Pentti, 1991, "Asymptotically efficient estimation of cointegration regressions," Econometric Theory 7, 1-21.

[38] Shimodaira, Hidetoshi, 2004, "Approximately unbiased tests of regions using multistep-multiscale bootstrap resampling," The Annals of Statistics 32, 2616-2641.

[39] Stock, James H., and Mark W. Watson, 1993, "A simple estimator of cointegrating vectors in higher order integrated systems," Econometrica 61, 783-820.

[40] Stone, Mervyn, 1974, "Cross-validation choice and assessment of statistical predictions," Journal of the Royal Statistical Society: Series B 36, 111-147.

[41] White, Halbert, 2000, "A reality check for data snooping," Econometrica 68, 10971126.

[42] Woodford, Michael, 2008, "How important is money in the conduct of monetary policy?" Journal of Money, Credit and Banking 40, 1561-1598.

[43] Zivot, Eric, and Donald W. K. Andrews, 1992, "Further evidence on the great crash, the oil-price shock, and the unit-root hypothesis," Journal of Business and Economic Statistics 20, 25-44. 
Table 1: Unit Root Tests with a One-time Break in the Drift of $m_{t}-p_{t}$ and $y_{t}$

\begin{tabular}{c|c|ccc|c}
\hline \hline & Zivot-Andrews Test & \multicolumn{2}{|c|}{ Banergee-Lumsdaine-Stock Test } & \multirow{2}{*}{ Perron Test } \\
\hline$m_{t}-p_{t}$ & -2.791 & $-7.626^{* *}$ & -0.113 & -2.573 & -2.963 \\
& $(1995: 7)$ & $(1990: 1)$ & $(1999: 3)$ & $(1995: 3)$ & $(1995: 5)$ \\
\hline$y_{t}$ & -2.273 & $-10.69^{* *}$ & 3.448 & -3.375 & -3.917 \\
& $(1987: 11)$ & $(1998: 8)$ & $(1999: 3)$ & $(1988: 10)$ & $(1987: 4)$ \\
\hline \hline $5 \%$ c.v. & -4.800 & -4.330 & -5.010 & -4.800 & -4.800 \\
\hline \hline
\end{tabular}

1. For each test, the null hypothesis is an integrated process with drift, and the relevant alternative hypothesis is a trend-stationary process with a one-time break in the trend at an unknown point in time.

2. Zivot and Andrew's (1992) test is conducted using Model (A), which allows for a one-time change in the level of the series.

3. For the recursive, rolling, and sequential tests of Banergee, Lumsdaine and Stock (1992), the mimimal Dicky-Fuller statistics is computed. The mimimal Dicky-Fuller statistics of the sequential test is computed from the mean-shift regressions.

4. Perron's (1997) test is conducted using the "crash" model, in which there is a shift in intercept.

5. Detected data points of shift are in parentheses. For the rolling test, a detected point of shift in parenthesis indicates the end of a rolling window.

6 . ** indicate the $5 \%$ level of significance. 
Table 2: Residual-based Tests for Cointegration with Regime Shifts

\begin{tabular}{ccc|ccc}
\hline \hline & Test Statistics & \multicolumn{3}{|c}{$5 \%$ c.v. } \\
& Semilog Log-log & & Bootstrap \\
Semilog & Log-log \\
\hline Inf-ADF & $-6.18^{* * *}-4.40$ & -5.50 & -5.60 & -5.60 \\
\hline Inf- $Z_{t}$ & $-6.12^{* * *}-5.06^{*}$ & -5.50 & -5.46 & -5.40 \\
\hline & & & & \\
\hline Inf- $Z_{\alpha}$ & $-58.71^{* * *}-47.39^{*}$ & -58.33 & -50.89 & -50.69 \\
\hline \hline
\end{tabular}

1. Tests are based on the regime shift model proposed by Gregory and Hansen (1996).

2. Asymptotic critical values are from Gregory and Hansen (1996).

3. Bootstrap critical values are computed from 5,000 replications under the null hypothesis of no cointegration.

4. For Inf-ADF, the lag length is selected using the $t$-test in Gregory and Hansen (1996).

5. * and *** indicate the $10 \%$ and $1 \%$ levels of significance for the bootstrap tests, respectively. 
Table 3: Tests for Parameter Instability of Money Demand Equations

\begin{tabular}{|c|c|c|c|c|c|}
\hline & \multirow{2}{*}{\multicolumn{2}{|c|}{ Test Statistics }} & \multicolumn{3}{|c|}{ 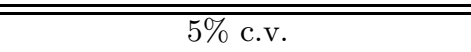 } \\
\hline & & & \multirow[t]{2}{*}{ Asymptotic } & \multicolumn{2}{|c|}{ Bootstrap } \\
\hline & Semilog & Log-log & & Semilog & Log-log \\
\hline \multicolumn{6}{|l|}{ Sup F } \\
\hline (1) & $\begin{array}{c}71.67^{* *} \\
(1995: 8)\end{array}$ & $\begin{array}{c}18.75 \\
(1995: 3)\end{array}$ & 17.3 & 48.95 & 37.91 \\
\hline (2) & $\begin{array}{c}20.75 \\
(1990: 9)\end{array}$ & $\begin{array}{c}16.08 \\
(1995: 3)\end{array}$ & 10.75 & 23.19 & 34.72 \\
\hline (3) & $\begin{array}{c}21.12 \\
(1990: 9)\end{array}$ & $\begin{array}{c}16.57 \\
(1995: 3)\end{array}$ & 10.71 & 25.19 & 35.23 \\
\hline (4) & $\begin{array}{c}48.37^{* * *} \\
(1995: 6)\end{array}$ & $\begin{array}{c}3.66 \\
(1997: 4) \\
\end{array}$ & 9.98 & 33.25 & 33.72 \\
\hline \multicolumn{6}{|l|}{ Mean F } \\
\hline (2) & 4.55 & 2.05 & 2.22 & 6.810 & 7.00 \\
\hline (3) & 4.65 & 2.15 & 2.14 & 6.810 & 7.15 \\
\hline (4) & 12.58 & 0.75 & 2.47 & 16.94 & 9.04 \\
\hline
\end{tabular}

1. Tests are based on the fully modified OLS proposed by Hansen (1992).

2. Asymptotic critical values from Kuo (1998) for a partial structural change and Hansen (1992) for a pure structural change.

3. Bootstrap critical values are from 5,000 replications under the null hypotheses of parameter constancy using the sieve bootstrap proposed by Chang et al. (2006).

4. In each panel, the first row, denoted (1), comprises tests of the entire cointegrating vector, the second row (2) gives tests of the intercept, the third row (3) gives tests of the coefficient on $y_{t}$, and the fourth row (4) gives tests of the coefficient on $i_{t}$

5. Data points with the largest F-statistics are in parentheses.

6. ** and $* * *$ indicate the $5 \%$ and $1 \%$ levels of significance for the bootstrap tests, respectively. 
Table 4: The Fluctuation and Nyblom Tests for Parameter Constancy of Long-run Money Demand Equations

\begin{tabular}{|c|c|c|c|c|c|}
\hline \multirow{3}{*}{ Tests } & \multirow{2}{*}{\multicolumn{2}{|c|}{ Test Statistics }} & \multicolumn{3}{|c|}{$5 \%$ c.v. } \\
\hline & & & \multirow[t]{2}{*}{ Asymptotic } & \multicolumn{2}{|c|}{ Bootstrap } \\
\hline & Semilog & Log-log & & Semilog & Log-log \\
\hline Fluctuation Test & $\begin{array}{c}0.777^{* *} \\
(1994: 12)\end{array}$ & $\begin{array}{c}0.497 \\
(1994: 5)\end{array}$ & 1.357 & 0.766 & 0.692 \\
\hline \multicolumn{6}{|l|}{ Nyblom Test } \\
\hline Sup Test & $\begin{array}{c}16.95^{\text {*** }} \\
(1995: 2)\end{array}$ & $\begin{array}{c}4.946 \\
(1995: 3)\end{array}$ & 2.400 & 12.44 & 7.756 \\
\hline Mean Test & $7.642^{* *}$ & 2.227 & 0.681 & 7.444 & 4.542 \\
\hline
\end{tabular}

1. The fluctuation test statistics are computed based on the largest eigenvalue $\lambda$ and the transformation $\xi=\log (\lambda /(1-\lambda))$, as proposed by Hansen and Johansen (1999).

2. The Nyblom tests statistics are computed based on a frst-order Taylor expansion of the score function as proposed by Hansen and Johansen (1999).

3. All test statistics are computed from the restricted-constant VAR models with three lags for the semilog model and seven lags for the log-log model. In compuatation of test statistics, cointegration rank of one is imposed on the restricted-constant VAR models.

4. The first-15 percent of the full sample are used as the base period.

5. Asymptotic and bootstrap critical values are from 5,000 simulations using Anders Warne's program Structural VAR 0.24. In bootstrapping, the block bootstrap procedure is performed with a block size of twelve.

6. Data points with the largest statistics are in parentheses for the fluctuation and Nyblom tests.

7. ** and $* * *$ indicate the $5 \%$ and $1 \%$ levels of significance for the bootstrap tests, respectively. 
Table 5: Parameter Estimates of Money Demand Equations

\begin{tabular}{|c|c|c|c|c|c|}
\hline Model & Period & 95\% C.I. & Constant & $y_{t}$ & $i_{t}$ \\
\hline Linear Semilog & 1985:7-1999:3 & $\begin{array}{l}\text { Asymptotic } \\
\text { Bootstrap }\end{array}$ & $\begin{array}{c}3.281 \\
(-2.367,8.819) \\
(-22.73,16.75)\end{array}$ & $\begin{array}{c}1.416 \\
(0.192,2.657) \\
(-1.487,7.502)\end{array}$ & $\begin{array}{c}-0.058 \\
(-0.094,-0.020) \\
(-0.249,0.030)\end{array}$ \\
\hline \multirow{2}{*}{ Joined Semilog } & 1985:7-1995:7 & $\begin{array}{c}\text { Asymptotic } \\
\text { Bootstrap }\end{array}$ & $\begin{array}{c}4.122 \\
(3.709,4.535) \\
(3.301,4.632) \\
\end{array}$ & $\begin{array}{c}1.202 \\
(1.109,1.295) \\
(1.089,1.390) \\
\end{array}$ & $\begin{array}{c}-0.037 \\
(-0.041,-0.033) \\
(-0.044,-0.032)\end{array}$ \\
\hline & 1995:8-1999:3 & $\begin{array}{l}\text { Asymptotic } \\
\text { Bootstrap }\end{array}$ & $\begin{array}{c}4.138 \\
(0.311,7.965) \\
(-20.24,5.607)\end{array}$ & $\begin{array}{c}1.325 \\
(0.472,2.177) \\
(1.106,6.722)\end{array}$ & $\begin{array}{c}-1.016 \\
(-1.293,-0.740) \\
(-2.120,-0.516)\end{array}$ \\
\hline Log-log & 1985:7-1999:3 & $\begin{array}{l}\text { Asymptotic } \\
\text { Bootstrap }\end{array}$ & $\begin{array}{c}5.832 \\
(4.716,6.928) \\
(3.998,9.338)\end{array}$ & $\begin{array}{c}0.839 \\
(0.594,1.081) \\
(0.072,1.243)\end{array}$ & $\begin{array}{c}-0.174 \\
(-0.190,-0.158) \\
(-0.239,-0.153)\end{array}$ \\
\hline
\end{tabular}

1. The estimation method employs the fully modified OLS proposed by Phillips and Hansen (1990).

2. $95 \%$ C.I. is the $95 \%$ confidence interval.

3. Asymptotic and bootstrap are the asymptotic and bootstrap confidence intervals, respectively. The bootstrap confidence intervals employ the sieve bootstrap proposed by Chang et al. (2006).

Table 6: Parameter Estimates of Money Demand Equations with Unitary Income Elasticity

\begin{tabular}{|c|c|c|c|c|c|}
\hline Model & Period & $95 \%$ C.I. & Constant & $y_{t}$ & $i_{t}$ \\
\hline Linear Semilog & 1985:7-1999:3 & $\begin{array}{c}\text { Asymptotic } \\
\text { Bootstrap }\end{array}$ & $\begin{array}{c}5.187 \\
(4.989,5.385) \\
(4.441,6.409)\end{array}$ & 1.000 & $\begin{array}{c}-0.068 \\
(-0.114,-0.022) \\
(-0.353,-0.001)\end{array}$ \\
\hline \multirow{2}{*}{ Joined Semilog } & 1985:7-1995:7 & $\begin{array}{l}\text { Asymptotic } \\
\text { Bootstrap }\end{array}$ & $\begin{array}{c}5.023 \\
(5.000,5.047) \\
(4.958,5.075)\end{array}$ & 1.000 & $\begin{array}{c}-0.035 \\
(-0.040,-0.030) \\
(-0.046,-0.026)\end{array}$ \\
\hline & 1995:8-1999:3 & $\begin{array}{l}\text { Asymptotic } \\
\text { Bootstrap }\end{array}$ & $\begin{array}{c}5.653 \\
(5.461,5.846) \\
(4.849,5.995)\end{array}$ & 1.000 & $\begin{array}{c}-1.092 \\
(-1.531,-0.653) \\
(-1.765,-0.403)\end{array}$ \\
\hline Log-log & 1985:7-1999:3 & $\begin{array}{l}\text { Asymptotic } \\
\text { Bootstrap }\end{array}$ & $\begin{array}{c}5.078 \\
(5.053,5.103) \\
(5.016,5.136)\end{array}$ & 1.000 & $\begin{array}{c}-0.161 \\
(-0.179,-0.143) \\
(-0.210,-0.140)\end{array}$ \\
\hline
\end{tabular}

1. See notes in Table 5 . 
Table 7: Performance Comparison of In-sample Predictability

\begin{tabular}{|c|c|c|c|c|c|c|c|}
\hline \multirow{2}{*}{ Period } & \multirow{2}{*}{ Method } & \multicolumn{3}{|c|}{$\begin{array}{l}\text { Sum of Squared Errors } \\
\text { (Bootstrap Probability) }\end{array}$} & \multirow{2}{*}{$\begin{array}{c}\text { Average } \\
i_{t}\end{array}$} & \multicolumn{2}{|c|}{ Average Interest Semielasticity } \\
\hline & & $\begin{array}{c}\text { Joined } \\
\text { Semilog }\end{array}$ & $\begin{array}{c}\text { Linear } \\
\text { Semilog }\end{array}$ & Log-log & & Joined Semilog & Log-log \\
\hline 1985:7-1999:3 & $\begin{array}{c}\text { In-sample } \\
\text { C.V. }\end{array}$ & $\begin{array}{c}0.407 \\
(0.429) \\
0.402 \\
(0.458) \\
\end{array}$ & $\begin{array}{c}+0.576 \\
(0.092) \\
+0.609 \\
(0.007) \\
\end{array}$ & $\begin{array}{c}-0.028 \\
(0.479) \\
-0.031 \\
(0.536) \\
\end{array}$ & 3.524 & $\begin{array}{c}-0.317 \\
(-0.558,-0.127)\end{array}$ & $\begin{array}{c}-0.158 \\
(-0.206,-0.137)\end{array}$ \\
\hline 1985:7-1995:7 & $\begin{array}{c}\text { In-sample } \\
\text { C.V. }\end{array}$ & $\begin{array}{c}0.079 \\
(0.560) \\
0.082 \\
(0.556) \\
\end{array}$ & $\begin{array}{c}+0.519 \\
(0.002) \\
+0.538 \\
(0.001) \\
\end{array}$ & $\begin{array}{c}+0.015 \\
(0.438) \\
+0.014 \\
(0.443) \\
\end{array}$ & 4.647 & $\begin{array}{c}-0.035 \\
(-0.040,-0.030)\end{array}$ & $\begin{array}{c}-0.043 \\
(-0.056,-0.037)\end{array}$ \\
\hline 1995:8-1999:3 & $\begin{array}{c}\text { In-sample } \\
\text { C.V. }\end{array}$ & $\begin{array}{c}0.328 \\
(0.437) \\
0.321 \\
(0.439) \\
\end{array}$ & $\begin{array}{c}+0.057 \\
(0.014) \\
+0.071 \\
(0.015) \\
\end{array}$ & $\begin{array}{c}-0.043 \\
(0.549) \\
-0.045 \\
(0.546)\end{array}$ & 0.437 & $\begin{array}{c}-1.092 \\
(-1.765,-0.403)\end{array}$ & $\begin{array}{c}-0.474 \\
(-0.617,-0.411)\end{array}$ \\
\hline
\end{tabular}

1. For the joined semilog model, the sum of squared errors (SSE) is reported. For the linear semilog and log-log models, the SSE difference with the joined semilog model is reported.

2. + indicates that the SSE of the linear semilog and log-log models is larger than that of the joined semilog model; - indicates the opposite.

3. The bootstrap probability is in parenthesis. The bootstrap probability is calculated using the sieve bootstrap method proposed by Chang et al. (2006) with 5,000 resamples.

4. C.V. indicates the leave-one-out cross-validation method proposed by Stone (1974).

5. Average $i_{t}$ indicates the sample average of overnight call rates.

6. Average interest semielasticity indicates the sample average of the estimated interest-rate semielasticity for the joined semilog model and the implied semielasticity for the log-log model.

7. The sample average of the bootstrap confidence interval for interest rate semielasticity is in parenthesis. The bootstrap confidence interval for the log-log model is obtained using the estimated interest-rate elasticity reported in Table 6 for the log-log model.

Table 8: Performance Comparison of Out-of-sample Predictability

\begin{tabular}{|c|c|c|c|c|c|c|}
\hline \multirow{2}{*}{ Period } & \multicolumn{3}{|c|}{$\begin{array}{l}\text { Sum of Squared Errors } \\
\text { (Bootstrap Probability) }\end{array}$} & \multirow{2}{*}{$\begin{array}{c}\text { Average } \\
i_{t}\end{array}$} & \multicolumn{2}{|c|}{ Average Interest Semielasticity } \\
\hline & $\begin{array}{l}\text { Jointed } \\
\text { Semilog }\end{array}$ & $\begin{array}{c}\text { Linear } \\
\text { Semilog }\end{array}$ & Log-log & & Jointed Semilog & Log-log \\
\hline 1999:4-2008:11 & $\begin{array}{c}14.82 \\
(0.795)\end{array}$ & $\begin{array}{l}+27.43 \\
(0.006)\end{array}$ & $\begin{array}{l}+1.448 \\
(0.199)\end{array}$ & 0.128 & $\begin{array}{c}-1.092 \\
(-1.765,-0.403)\end{array}$ & $\begin{array}{c}-65.85 \\
(-85.89,-57.26)\end{array}$ \\
\hline 1999:4-2006:6 & $\begin{array}{c}4.128 \\
(0.873)\end{array}$ & $\begin{array}{l}+26.46 \\
(0.000)\end{array}$ & $\begin{array}{l}+1.561 \\
(0.127)\end{array}$ & 0.027 & $\begin{array}{c}-1.092 \\
(-1.765,-0.403)\end{array}$ & $\begin{array}{c}-87.66 \\
(-114.3,-76.22)\end{array}$ \\
\hline 2006:7-2008:11 & $\begin{array}{c}10.69 \\
(0.373) \\
\end{array}$ & $\begin{array}{l}+0.962 \\
(0.077) \\
\end{array}$ & $\begin{array}{l}-0.113 \\
(0.550) \\
\end{array}$ & 0.429 & $\begin{array}{c}-1.092 \\
(-1.765,-0.403) \\
\end{array}$ & $\begin{array}{c}-0.420 \\
(-0.548,-0.365) \\
\end{array}$ \\
\hline
\end{tabular}

1. See notes in Table 7 . 


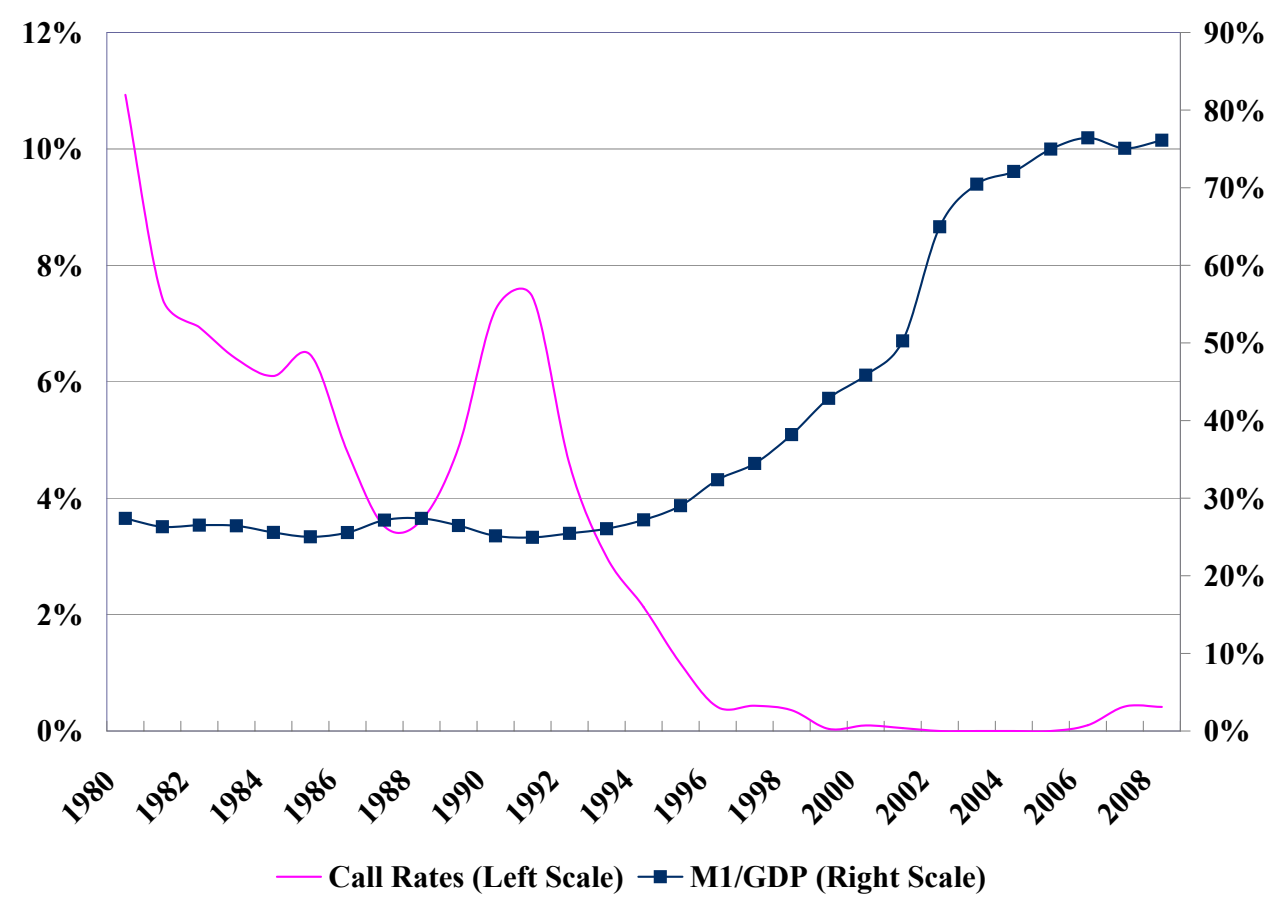

Figure 1. Overnight Call Rates and Nominal M1 Stock 


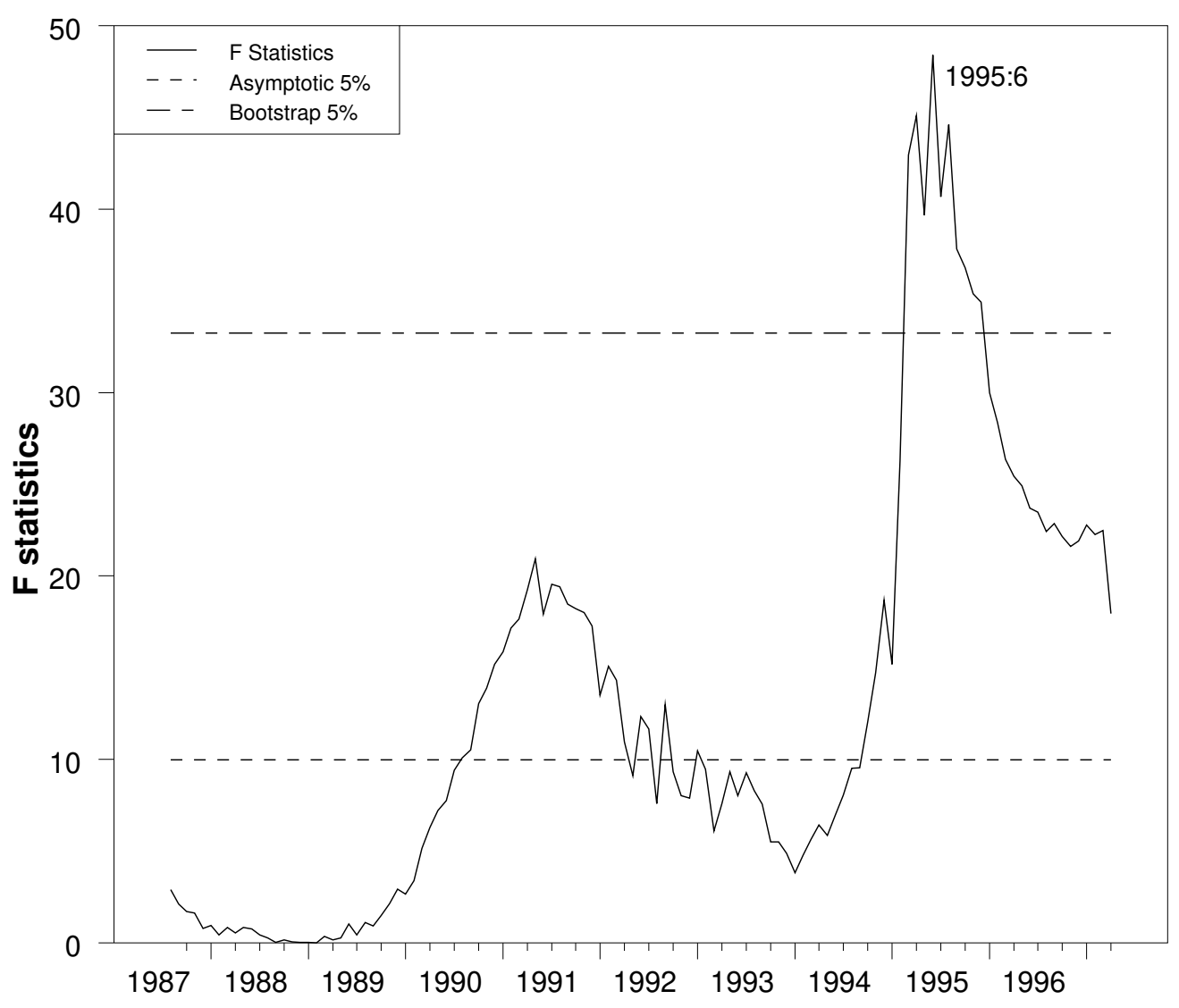

Figure 2. Testing Partial Structural Breaks for Interest Rate Semielasticity 


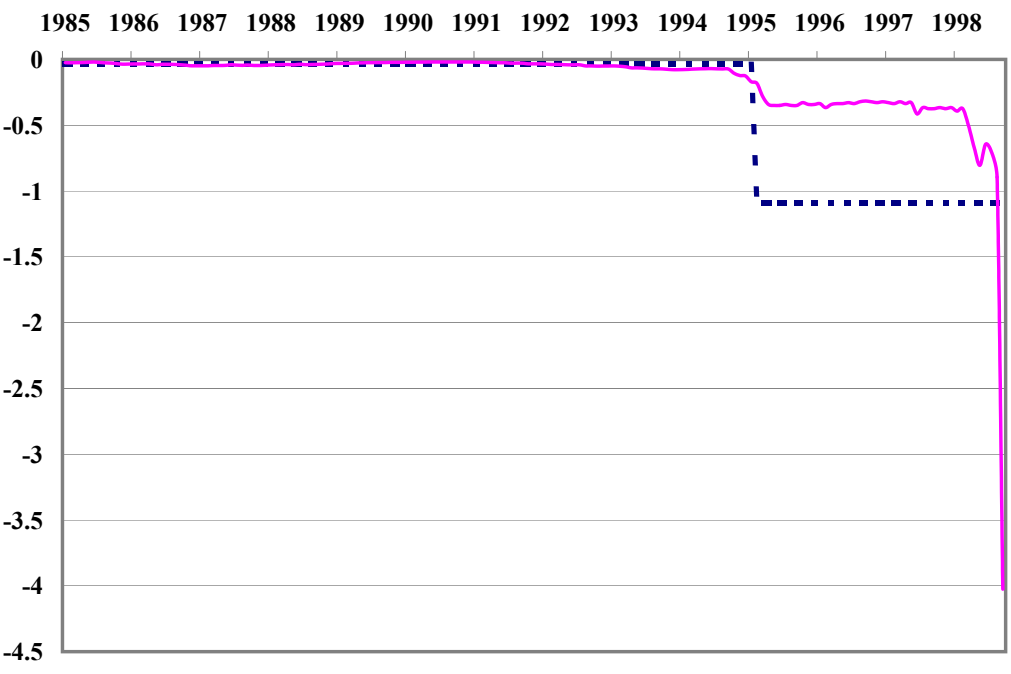

- - Semi-elasticity (Jointed Semi-log Model) Implied Semi-elasticity (Log-log Model)

Figure 3. Interest Rate Semielasticity for In-sample Period

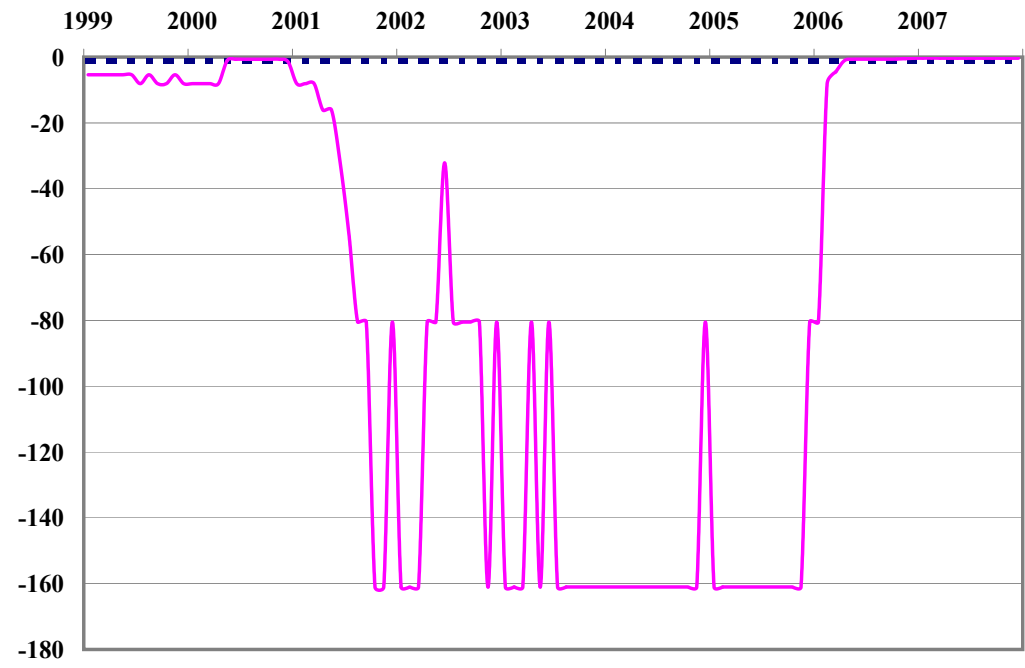

- - Semi-elasticity (Jointed Semi-log Model) — Implied Semi-elasticity (Log-log Model)

Figure 4. Interest Rate Semielasticity for Out-of-sample Period 